\title{
Evaluation of RFID Technology to Capture Surgeon Arrival Time to Meet American College of Surgeons Committee on Trauma Verification Guidelines
}

\author{
Heather Lyu ${ }^{1,2}$ Nicholas Faoro ${ }^{3}$ Meghan McDonald ${ }^{3}$ Molly Jarman ${ }^{4}$ Kevin Kreitzman ${ }^{5}$ \\ Ali Salim ${ }^{1,2,3}$ Adam Landman ${ }^{2,6}$
}

${ }^{1}$ Department of Surgery, Brigham and Women's Hospital, Boston, Massachusetts, United States

${ }^{2}$ Harvard Medical School, Boston, Massachusetts, United States

${ }^{3}$ Division of Trauma, Burn, Surgical Critical Care, and Emergency

Address for correspondence Heather Lyu, MD, Department of Surgery, Brigham and Women's Hospital, 75 Francis Street, Boston, General Surgery, Brigham and Women's Hospital, Boston, Massachusetts, United States

${ }^{4}$ Center for Surgery and Public Health, Brigham and Women's Hospital, Boston, Massachusetts, United States

${ }^{5}$ Clinical Engineering, Brigham and Women's Hospital, Boston, Massachusetts, United States

${ }^{6}$ Department of Emergency Medicine, Brigham and Women's Hospital, Boston, Massachusetts, United States

ACI Open 2019;3:e13-e17.

\section{Abstract}

Keywords

- RFID

- quality improvement

- trauma verification

- automation
Background The presence of an attending surgeon at all highest-level trauma activations is a requirement for American College of Surgeons-Committee on Trauma (ACS-COT) verification for level I to III trauma centers. Programs must demonstrate compliance with this criterion at least $80 \%$ of the time. Documentation of compliance can increase administrative burden presenting an opportunity for automation.

Objectives The aim of this quality improvement project was to compare surgeon arrival documentation rates obtained utilizing radio-frequency identification (RFID) technology with manual documentation.

Methods This project was conducted at a single level-I trauma center. RFID badges were distributed to all trauma surgeons. Arrival times for surgeons using manual nursing documentation and RFID activation were collected from October 2017 through March 2018. Presence of appropriate documentation and attending arrival within 15 minutes of trauma activation were compared by documentation method: nursing manual documentation or RFID system.

Results There were 98 code trauma activations included in the analysis over the 6month period. Nursing documentation of trauma surgeon attendance occurred $83 \%$ of the time $(n=81)$, with $81 \%(n=79)$ in compliance within 15 minutes of code trauma activation. RFID badges were activated $91 \%(n=89)$ of the time, with $86 \%$ $(n=84)$ in compliance within 15 minutes. There was no statistically significant difference between the rates of nursing documentation and RFID badge activation. Conclusion RFID technology is a reliable, complementary method of documenting compliance for trauma surgeon attendance. Trauma centers searching for technological solutions to address compliance with ACS-COT guidelines and to reduce administrative burden may consider the use of RFID technology. received

September 5, 2018 accepted after revision January 10, 2019
DOI https://doi.org/ 10.1055/s-0039-1684003. ISSN 2566-9346. (c) 2019 Georg Thieme Verlag KG Stuttgart · New York
License terms

() (1) 


\section{Background and Significance}

The American College of Surgeons (ACS) Committee on Trauma (COT) describes guidelines and requirements for the resources for trauma care at various levels of acuity. ${ }^{1}$ One of the wellknown requirements of ACS-COTverification is the presence of an attending surgeon at all highest-level trauma activations with a maximum acceptable response time of 15 minutes for level I and II trauma centers, and 30 minutes for level II centers. ${ }^{1}$ The program must demonstrate compliance with this criterion at least $80 \%$ of the time. ${ }^{1}$ Despite this requirement, many trauma centers struggle to accurately document the trauma attending surgeon's presence.

Brigham and Women's Hospital (BWH) has been a verified level I trauma center since 1993. As part of the verification requirements, the hospital has kept consistent documentation of an attending surgeon's arrival in response to a trauma activation with $90 \%$ compliance. Trauma attending arrival times were previously recorded by emergency department nurses on a paper trauma flow sheet. Trauma flow sheets serve as documentation of all providers present during the trauma activation. In high-level activations with a severely injured patient, a team should include the following: (1) a general surgeon, (2) an emergency physician, (3) surgical and emergency residents, (4) emergency department nurses, (5) a laboratory technician, (6) a radiology technologist, (7) a critical care nurse, (8) an anesthesiologist, (9) an operating room nurse, (10) security officers, (11) a chaplain or social worker, and (12) a scribe. ${ }^{1}$ In 2015 , the hospital transitioned to a new enterprise electronic medical record system. During this transition, the documentation of trauma surgeon attendance dropped to $40 \%$. Despite trauma surgeons remaining in-house while on call, there was no clear documentation of their arrival at many trauma activations and the dramatic drop in reported attending surgeon attendance was thought to be secondary to a new and complex electronic documentation process for the trauma nurses. Electronic documentation required clicking through multiple pages during a typically chaotic and medically challenging time period. Since this initial drop, nursing documentation during trauma activations underwent multiple process improvements to increase documentation of attending response time. However, an innovative and more automated system was sought to accurately document trauma surgeons' compliance with ACS guidelines given the administrative burden to nursing staff.

Radio-frequency identification (RFID) technology uses radio-frequency electromagnetic fields for storing and retrieving data. The RFID technology has been used in health care systems for various purposes including tracking patients and medical personnel, medical devices, and medications. $^{2}$ However, the utilization of RFID technology to automate documentation required for ACS-COT verification has not been described in the literature. Since RFID tags can be well suited to automatically detect a person's location and time of arrival, they might be able to accurately document trauma team presence during trauma activations.

\section{Objectives}

Our trauma program gave RFID badges to trauma team attending physicians in an attempt to determine its utility in effortlessly tracking trauma attending presence. We aimed to compare attending physician arrival documentation rates obtained through the RFID system with manual documentation by nurses to better understand the utility of RFID technology for tracking trauma team members.

\section{Methods}

This quality improvement project was conducted at $\mathrm{BWH}$, a level I trauma center, in Boston, Massachusetts. RFID antennas and beacons already existed in multiple locations throughout the emergency department and were being used for medical device and equipment tracking. Eleven beacons in the primary trauma bays were upgraded and configured to detect the signals from the RFID badges distributed to trauma surgeons ( - Fig. 1). The two major trauma rooms were further outfitted with infrared technology that allowed localization of the badges.

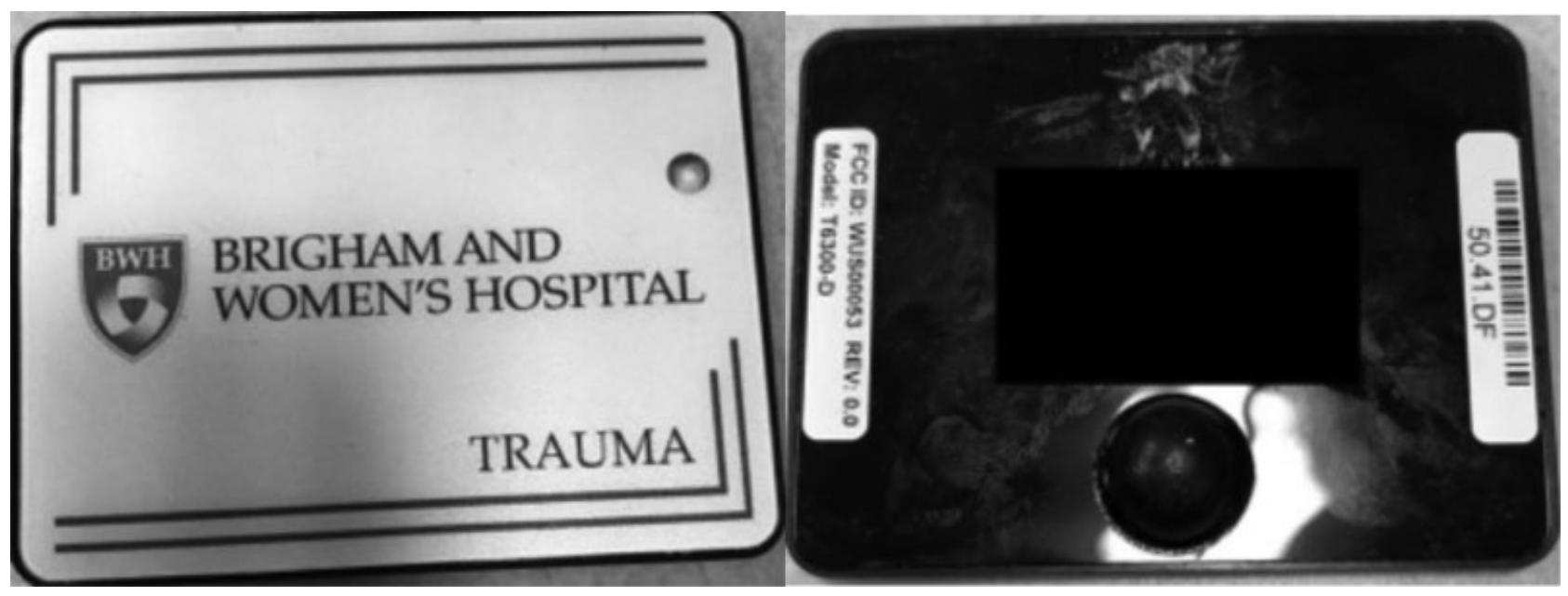

Fig. 1 Photos of the RFID badge distributed to trauma surgeons. 
A pilot was initially conducted with 3 RFID badges and 11 trauma surgeons to confirm that the RFID system was accurately detecting arrival in the trauma bays. Following the success of this pilot, individual RFID badges were distributed to all 11 attending trauma surgeons in October 2017. The trauma surgeons were asked to wear their RFID badges when they were on call.

The total number of code traumas, the highest level of trauma activation at $\mathrm{BWH}$, was recorded. Arrival times for each trauma surgeon were documented using two separate methodologies: nursing documentation of surgeon arrival to the trauma bay and arrival time captured and recorded by the RFID beacons. Data were collected from October 3, 2017 through March 25, 2018. There were two occurrences of planned RFID system downtimes for upgrades. Any trauma activations that occurred during these two time periods were excluded from the study.

The primary outcomes were the percent of attending physician documentation for code trauma activations and the percent of documentation of attending physician arrival time within 15 minutes. We assessed whether attending physician attendance not recorded by the RFID system or nursing manual documentation was captured by the other system. Using attending arrival time stamps, we also compared the time differences between RFID and nursing documentation of attending physician arrival. This was a quality improvement project within the Division of Trauma, Burn, Surgical Critical Care, and Emergency General Surgery.

\section{Statistical Analysis}

Trauma attending documentation percent and arrival time percent were compared using Pearson's chi-square test for independence. The mean absolute error was calculated to assess the time difference of attending physician arrival documentation by nursing staff and RFID badge activation. Statistical analysis was performed using R (R Foundation for Statistical Computing, Vienna, Austria) and statistical significance was defined as $p<0.05$.

\section{Results}

There was a total of 107 code trauma activations over the 6month period from October 2017 through March 2018. Nine code trauma activations that occurred during planned downtimes for RFID system maintenance and upgrades were excluded from analysis, leaving 98 trauma activations.

Nursing documentation of trauma surgeon attendance occurred $83 \%$ of the time $(n=81)$, with $81 \%(n=79)$ arriv- ing within 15 minutes of code trauma activation. RFID badges were activated $91 \%(n=89)$ of the time. Trauma attending arrival was in compliance within 15 minutes of code trauma activation $86 \%$ ( $n=84$ ) of the time. There was no statistically significant difference between the percent documentations of the nurses and the RFID system ( - Table 1 ).

Out of the 17 occurrences in which nurses did not document surgeon arrival time, $88 \%(n=15)$ were appropriately captured by RFID badge activation. Of the nine trauma activations without RFID badge detection, two (22.2\%) also did not have nursing documentation of surgeon arrival. The remaining seven (77.8\%) trauma cases without RFID badge activation were otherwise documented by nursing staff.

Time recorded by the RFID system between trauma activation and attending arrival ranged from -24 minutes to 72 minutes with a mean of 3.4 minutes. Time recorded by nursing documentation between trauma activation and attending arrival ranged from -25 minutes to 19 minutes with a mean of 1.9 minutes. Negative durations were due to attending physicians' presence in the trauma bay prior to the primary trauma activation. The mean absolute error calculated for nursing time documentation as compared with the time of RFID badge activation was 7.1 minutes.

\section{Discussion}

Our study shows that it is feasible to use RFID technology to accurately track trauma surgeon compliance with ACS-COT guidelines for level I trauma centers. While RFID badge activation proved to document compliance at a higher rate than nursing documentation, the differences in documentation rates by nurses and RFID badge activation were not statistically significant. Moreover, RFID badge activation is not comprehensive. A small number of attending physician arrivals missed by RFID technology were captured by nursing documentation.

ACS verification, while not required, has demonstrated benefits for many trauma centers with a significant impact on patient outcomes and cost savings. ${ }^{3}$ Trauma centers seeking ACS-COT verification have been on the rise as studies have shown that verified centers have significantly improved outcomes for specific injuries including severe traumatic brain injury and acute respiratory distress syndrome ${ }^{4-7}$ and have significant reduction in complications for almost all trauma patients. ${ }^{8}$ While documentation of compliance of trauma surgeon response to an activation is only one component of the process of verification, the lack thereof can threaten a single institution's status. ${ }^{1}$ Specifically, this criteria is considered a type I criteria or deficiency, of which there are 61 in

Table 1 Documentation of trauma surgeon arrival at code trauma activations ( $N=98$ trauma activations)

\begin{tabular}{|l|l|l|l|l|}
\hline $\begin{array}{l}\text { Documentation } \\
\text { method }\end{array}$ & \multicolumn{2}{|l|}{$\begin{array}{l}\text { Trauma activations with attending physician } \\
\text { documentation } \\
N(\%)\end{array}$} & $\begin{array}{l}\text { Trauma activations with attending physician } \\
\text { arrival within 15 minutes } \\
N(\%)\end{array}$ \\
\hline $\begin{array}{l}\text { Nursing } \\
\text { documentation }\end{array}$ & $81(83 \%)$ & $\begin{array}{l}\mathrm{X}^{2}=2.84 \\
p=0.09\end{array}$ & $79(81 \%)$ & $\begin{array}{l}\mathrm{X}^{2}=0.91 \\
\mathrm{p}=0.3\end{array}$ \\
\hline RFID system & $89(91 \%)$ & & $84(86 \%)$ & \\
\hline
\end{tabular}

Abbreviation: RFID, radio-frequency identification. 
total; trauma centers seeking verification or reverification must meet all type I criteria at the time of the review to receive ACS-COT certification. ${ }^{1}$ Hospitals with any type I deficiency at the time of review cannot be verified and must undergo another site visit within 6 to 12 months to demonstration correction. ${ }^{1}$ The potential loss of benefits associated with ACS-COT verification including both improved quality of patient care and reduced costs could be significant.

Our results confirm that nursing staff are capable of appropriately documenting trauma surgeon attendance and meeting criteria for ACS verification. Nevertheless, with the integration of technology in hospital systems and daily patient care, there could be less reliance on manual documentation particularly during chaotic clinical situations such as trauma activations and mass casualty events. RFID badge activation can provide objective metrics for documentation that are less vulnerable to human error. In addition, use of RFID technology could accurately document the arrival times for subspecialty surgeons, particularly orthopedic and neurosurgery, who are now required to respond within 30 minutes for various indications at level I and II trauma centers. This is an issue that has proven to be challenging for many trauma centers. New technologies have great potential to enhance resource and personnel management during these challenging situations.

RFID technology has been described as one of the most promising technologies due to its versatility, easy integration into health care systems, and need for minimal human intervention and interference. ${ }^{2}$ Also, RFID does not require line-ofsight like traditional barcodes and can read multiple tags simultaneously with a longer read range. ${ }^{2,9,10}$ RFID systems have been studied extensively in the setting of equipment tracking. At Beth Israel Deaconess Medical Center, RFID technology is used to track equipment and patient beds. ${ }^{10}$ The use of RFID tracking for medical devices and equipment has been demonstrated to contribute to significant cost savings and to the reduction of theft loss and unnecessary waste. ${ }^{10}$

In a study by Parlak et al, RFID technology was used at Children's National Medical Center (CNMC) during trauma activations to address the multiple challenges associated with the fast pace, high risk, and crowded nature of a trauma bay. ${ }^{2}$ This study used RFID technology to track important objects and tasks during traumas to ensure they were utilized and completed correctly. In addition, RFID tags were placed on eight team members (team leader, physician, anesthesiologist, primary nurse, scribe, medication nurse, respiratory therapist, and technician) involved in the trauma to provide further evidence that certain tasks were completed. This study proved the feasibility of RFID technology to detect and track not only objects but also providers in a time critical medical setting.

RFID technology has also been utilized for patient tracking. In the aforementioned study, RFID use was expanded to track patients including neonatal intensive care unit babies. ${ }^{11}$ This technology for tracking patients has been shown to improve the accuracy of patient identification ${ }^{10,12}$ as well as decrease delay in patient progression through multiple phases of case within their hospitalization or outpatient care.

RFID utilization and integration has proven to be beneficial in multiple care settings. The intent of this project was to show that compliance rates with RFID badge activations are equivalent to or better than that of nursing documentation. Our results show that RFID badge activations are as reliable as current documentation methods. The utility of RFID technology demonstrated in our project is its ability to supplement nursing documentation and subsequently reduce administrative burden, allowing nurses to focus on more patient care critical tasks during trauma activations.

Our study was successful for several reasons. There are 11 trauma surgeons in the department making it relatively easy to closely monitor for compliance with the RFID badges as well as the ACS-COT guidelines. Also, the isolation of trauma bays to a small area of the emergency department allowed for a more accurate reading of the RFID badges. Similar accuracy could be obtained in a larger area by deploying more infrared readers.

Finally, the RFID technology was already being utilized at BWH for other purposes and upgrades of the existing beacons did not require significant changes to the institution's current real-time locating system and management. Trauma surgeons were asked to identify potential factors behind lack of RFID badge activation at nine separate trauma activations. The only stated reason for noncompliance with wearing the RFID badges included a lost or missing badge.

A major limitation of the integration of any new technology is that all systems, including RFID systems, undergo periodic updates and require maintenance that can lead to downtime. Alternative documentation methods during maintenance periods need to be addressed. In our quality improvement project, all planned downtimes were precluded by multiple methods of communication to make the entire trauma team aware and to ensure continued documentation by nurses. Importantly, this project lacked a gold standard comparison, such as independent observer recording of attending arrival time, preventing us from determining which arrival time documentation method is superior. As with any technology, there may be security and privacy concerns with the utilization of RFID badges. Users may perceive RFID badges as a violation of their privacy as their movements within the hospital could be tracked. Also, we did not evaluate the cost implications of implementing RFID technology which could be considerable, particularly for institutions without existing RFID beacons. Finally, there was no formal qualitative study on reasons behind missing RFID badges or surgeon and trauma team satisfaction with the technology. Future studies can include a cost analysis as well as a qualitative component to describe the trauma team perception of team communication after implementation and the potential impact of the RFID technology on trauma outcomes.

\section{Conclusion}

Since RFID technology has proven to be a reliable, complementary method of documenting compliance for trauma surgeon attendance, we hope to eventually replace nurse documentation of trauma surgeon compliance. Other level I and II trauma centers searching for technological solutions to 
address compliance with ACS-COT guidelines should consider the use of RFID systems.

\section{Clinical Relevance Statement}

This manuscript concisely discusses our findings of a novel and innovative method to track the presence of trauma surgeons at the highest-level trauma activations. We used RFID technology to capture surgeon arrival time at a single level I trauma center and found that it is equivalent, if not superior, to our current methodology, which is often burdensome to the trauma nursing staff and the surgical residents. We believe that using RFID technology could be more efficient and accurate and reduce administrative burden for trauma teams allowing all providers to focus on more timesensitive and critical patient care tasks.

\section{Protection of Human and Animal Subjects}

This study did not involve any human subjects. Data were collected as part of a quality improvement project and did not require review by the institutional review board.

\section{Funding}

Dr. Lyu was supported by the National Library of Medicine Institutional training grant for research training in biomedical informatics and data science (T15) under award number T15LM007092.

\section{Conflict of Interest}

None declared.

\section{Acknowledgment}

We thank Lorraine Fenelon from the BWH information systems team for managing the implementation of the RFID system.

\section{References}

1 Committee on Trauma; American College of Surgeons. Resources for Optimal Care of the Injured Patient 2014. Available at: https:// www.facs.org/ /media/files/quality\%20programs/trauma/vrc\% 20resources/resources\%20for\%20optimal\%20care.ashx. Accessed February 11, 2019

2 Parlak S, Sarcevic A, Marsic I, Burd RS. Introducing RFID technology in dynamic and time-critical medical settings: requirements and challenges. J Biomed Inform 2012;45(05):958-974

3 Choi PM, Hong C, Woods S, Warner BW, Keller MS. Early impact of American College of Surgeons-verification at a level-1 pediatric trauma center. J Pediatr Surg 2016;51(06):1026-1029

4 Brown JB, Watson GA, Forsythe RM, et al. American College of Surgeons trauma center verification versus state designation: are Level II centers slipping through the cracks? J Trauma Acute Care Surg 2013;75(01):44-49

5 Norwood S, Cook AD, Berne JD. Level I verification is associated with a decreased mortality rate after major torso vascular injuries. Am Surg 2011;77(01):32-37

6 DuBose JJ, Browder T, Inaba K, Teixeira PG, Chan LS, Demetriades D. Effect of trauma center designation on outcome in patients with severe traumatic brain injury. Arch Surg 2008;143(12):1213-1217

7 Recinos G, Dubose J, Teixeira PG, et al. ACS trauma centre designation and outcomes of post-traumatic ARDS: NTDB analysis and implications for trauma quality improvement. Injury 2009;40(08):856-859

8 Grossman MD, Yelon JA, Szydiak L. Effect of American College of Surgeons Trauma Center designation on outcomes: measurable benefit at the extremes of age and injury. J Am Coll Surg 2017;225 (02):194-199

9 Kolokathi A, Rallis P. Radio frequency identification (RFID) in healthcare: a literature review. Stud Health Technol Inform 2013; 190:157-159

10 Yao W, Chu CH, Li Z. The adoption and implementation of RFID technologies in healthcare: a literature review. J Med Syst 2012; 36(06):3507-3525

11 Wicks AM, Visich JK, Li S. Radio frequency identification applications in hospital environments. Hosp Top 2006;84(03):3-8

12 Cangialosi A, Monaly JE, Yang SC. Leveraging RFID in hospitals: patient life cycle and mobility perspectives. IEEE Commun Mag 2007;45(09):18-23 